\title{
Predicting Difficulty Score for Spinal Anesthesia in Transurethral Lithotripsy Surgery
}

\author{
Hossein Khoshrang ${ }^{1}$; Siavash Falahatkar ${ }^{1,}$; Abtin Heidarzadeh ${ }^{1}$; Mohsen Abad ${ }^{1}$; Nadia \\ Rastjou Herfeh ${ }^{1}$; Bahram Naderi Nabi ${ }^{1}$ \\ ${ }^{1}$ Urology Research Center, School of Medicine, Guilan University of Medical Sciences, Rasht, Iran \\ ${ }^{*}$ Corresponding author: Siavash Falahatkar, Urology Research Center, School of Medicine, Guilan University of Medical Sciences, Rasht, Iran. Tel/Fax: $+98-1315525259$, E-mail: \\ falahatkar_s@yahoo.com
}

Received: November 25, 2013; Revised: January 12, 2014; Accepted: April 21, 2014

\begin{abstract}
Background:Spinal anesthesia (SA) is the most common regional anesthesia (RA) conducted for many surgical procedures.
Objectives: The current study aimed to predict the difficulty score of SA, by which to reduce the complications and ultimately improve the anesthesia quality.

Materials and Methods: Transurethral Lithotripsy (TUL) surgery candidates were enrolled in this observational study from 2010 to 2011. Before SA, the patient`s demographic information along with the Body Mass Index (BMI), lumbar spinous process status, spinal deformity, radiological signs of lumbar vertebrae, and a history of spinal surgery or difficult SA were recorded, then the patients underwent SA in L3-L4 interspinous process space. Information about Cerebrospinal Fluid (CSF) visibility at the first attempt (easy SA) and the times of trying with shifting in that space or trying the second space (moderate SA) and the third space (difficult SA) were recorded. Multinominal regression and relative operating characteristic (ROC) curve were used for statistical analysis.

Results: Hundred and one patients were enrolled. Of these patients, 50 (49.5\%) underwent SA by the first attempt of the first space, in 36 patients (35.6\%) it was moderate and in 15 patients (14.9\%) it was difficult. There was no significant relationship between difficulty score of SA and gender, age, height, and history of previous difficult SA. But there was a significant relationship between difficulty score of SA and lumbar spinous process status $(\mathrm{P}=0.0001)$, radiological profile of the lumbar spine $(\mathrm{P}=0.0001)$, the status of lumbar deformity $(\mathrm{P}=$ $0.007)$, and $\mathrm{BMI}(\mathrm{P}=0.006)$. Then using the ROC curve to predict the difficult SA, the cutoff point was 8.5 with $86.7 \%$ and $86 \%$ sensitivity and specificity, respectively.

Conclusions: It seems that considering the clinical examination of patients before SA focusing on lumbar spinous process status, presence of lumbar deformity, calculation of BMI and radiological signs of lumbar vertebrae can be helpful in predicting SA difficulty.
\end{abstract}

Keywords: Spinal Anesthesia; Lithotripsy; Severity Score

\section{Background}

Regional anesthesia (RA) is the first choice for lower abdominal, lower extremity, urologic, gynecologic, and anorectal surgery (1). The RA is tremendously safe, has multiple benefits, and can be compared with general anesthesia (GA), these advantages are consisted of reduction in morbidity and mortality, superior pre- and postoperative analgesia and it is economical (2). Spinal anesthesia (SA) is the most common RA conducted for many surgical procedures. In SA the rates of venous thromboembolism, myocardial infarction, requirements to postoperative analgesia, sympathetic response to surgical stimulation and several other complications are less (3). Among the lithotripsy methods, transurethral lithotripsy (TUL) is a very appropriate method. It is safe, with few complications for ureteral stones, and the first-selected method in the ureteral stones (4-6). Many conditions such as osteoarthritis, ankylosing spondylitis, kyphoscoliosis, previous spinal surgery, and degenerative disc diseases with the intervertebral space collapse may cause problems in the needle access (7). Multiple attempts at needle placement may cause patients' discomfort and higher incidence of spinal hematoma, postdural puncture headache (PDPH) and trauma to neural structures (8). Thus as SA is widely applied, a clear grading system to evaluate the probability of a difficult neuroaxial block is vital and important. It can assist to reduce the incidence of multiple attempts rendering the technique more acceptable and less risky (9). The failure rate of spinal anesthesia was reported from $<1 \%$ to $17 \%(9,10)$, and the average failure rate was in the range of $11.6 \%(9,11)$.

\section{Objectives}

The current study was designed prospectively to identify the predictive factors of SA difficulty in patients with Transurethral Lithotripsy.

Copyright (C) 2014, Iranian Society of Regional Anesthesia and Pain Medicine (ISRAPM); Published by Kowsar. This is an open-access article distributed under the terms of the Creative Commons Attribution-NonCommercial 4.0 International License (http://creativecommons.org/licenses/by-nc/4.0/) which permits copy and redistribute the material just in noncommercial usages, provided the original work is properly cited. 


\section{Materials and Methods}

Following the approval of Research Ethics Committee of Guilan University of Medical Sciences and taking the informed consent from the participants, an analytical cross sectional study was performed on patients aged 15 to 75 years old undergoing TUL based on American Society of Anesthesiology Classification (ASAI, II) from 2010 to 2011. Exclusion criteria were contraindication to spinal anesthesia such as infection in the injection site, intrinsic or idiopathic coagulopathy, bacteremia, raised Intracranial Pressure (ICP), patients' disagreement to take part in the experiment and patients' mental imbalance. The current study complied with ethical conditions, and informed consents were taken from 101 patients who were candidates for elective TUL. Before beginning SA, the senior assistant advisor recorded the patients' information such as age, gender, weight, height, body mass index (BMI), anatomy of spinous process on the questionnaire, and spinous process was divided into three categories: visible, invisible but palpable, invisible and impalpable. Spinal deformity was measured by the degree of curvature of spine in axial plane or rotation from sagittal plane (lordosis, kyphosis and scoliosis). In Kidney, Ureter, Bladder $\mathrm{X}$-ray (KUB) radiography, radiographic features of spine determined difficulty characteristics (osteophites, ligament calcification, reduced intervertebral space), a history of previous difficult SA or epidural anesthesia, and a history of surgery on lumbar spine were also recorded. Patients with the inclusion criteria underwent TUL with SA by an anesthesiologist with more than 10 years of experience. SA was performed in all patients in the sitting position by median approach using $25 \mathrm{G}$ spinal needle (Whitacre by Nesco) $2 \mathrm{~mL}$ of 5\% lidocaine and epinephrine $(0.2 \mathrm{mg})$ were injected in L3-L4 interspinous process space. Before anesthesia, for all of the patients, intravascular hydration by 5-7 mL/kg IV dextrose free balanced salt solution was done.

Information about Cerebrospinal Fluid (CSF) visibility in the first attempt (easy SA) and the number of trying times with shifting in that space or the second space trying (moderate SA) and trying the third space (difficult SA) were recorded. If there were a need of more than three consecutive attempts or extra analgesic drugs and other anesthetic techniques, it would be considered as a SA failure (impossible SA application) or incomplete SA (replacing general anesthesia in the case of the patient's agitation, pain and no tolerance of SA). Data was analyzed by STATA software version 10.0. Chi-square and one way ANOVA test were used for preliminary analysis, then multinomial regression was used to evaluate the correlation of SA difficulty score for quantitative variables, and Spearman's rho test was used to rank order qualitative variables. The variables with significant relationship were modeled by logistic regression method. Relative Operating Characteristic (ROC) curve was used and the cutoff point was set to maximum under curve surface to assess the accuracy of quantitative and qualitative factors which had significant correlation. First, the mean age, weight, and the quantitative variables in different groups of patients were compared; then the severity of spinal anesthesia was assessed by ANOVA. Post hoc test was used to compare the differences in different groups. But eventually due to the age group, BMI, height, weight deletion, the final comparison was done by Chi-square.

\section{Results}

Gender combination in 101 patients in the current study was 72 males (71.3\%) and 29 females (28.7\%); all demographic information is presented in Table 1.SA was successful and easy in the first attempt in 50 patients (49.5\%), in 36 cases (35.6\%) the difficulty score for SA was moderate and 15 cases (14.9\%) had a difficult SA. (Table 2) One patient had a history of spinal surgery and 15 patients $(14.9 \%)$ had the history of difficult spinal or epidural anesthesia. Also the history of previous difficult spinal or epidural anesthesia did not influence the difficulty score of $S A(P=0.39)$. The one way ANOVA square test showed significant correlation between spinous process condition and the difficulty of SA, parametric test showed no significant difference between the mean of weight and the spinal severity $(\mathrm{P}=0.07)$, and also Chisquare test showed the same results $(P=0.05)$. In the preoperative examination spinous process, the lumbar spine was prominently visible in 38 patients (37.6\%), in 44 patients $(43.6 \%)$ spinous process was not visible, but probably palpable, and in 19 patients $(18.8 \%)$ it was neither visible nor palpable (Table 3$)(\mathrm{P}<0.01$, chi $=41.06)$. Among the 101 patients, 81 patients (78.6\%) had no spinal deformity and 20 patients $(19.8 \%)$ had skeletal spinal deformity; out of these, six cases (5.8\%) had kyphosis, 11 cases (10.7\%) had scoliosis and five patients (4.9\%) had lordosis. Among the 20 patients, two had both skeletal spinal deformity (kyphosis, scoliosis or lordosis) simultaneously. Chi-square test showed significant correlation between spinal deformity and difficulty score of SA $(\mathrm{P}=0.007$, chi $=9.97)$. According to BMI, patients were divided into four groups (Table 4). The parametric test of the one way ANOVA showed significant correlation between BMI and difficulty score of SA $(\mathrm{P}<0.01)$, Spearman's rho correlation coefficient confirmed this result $(\mathrm{P}<0.01, \mathrm{R}=0.286)$, which meant a correlation between the increased BMI and increased difficulty score of SA. Radiologic features of lumbar vertebra in KUB in 50 patients (49.5\%) were normal, and 51 patients (50.5\%) had lesions in KUB; from this group, 10 cases $(8.65 \%)$ had ligament calcifications, 17 patients $(14.7 \%)$ had osteophites, and in 34 patients (33.6\%) vertebral space had reduced. Among the patients, 13 cases had just one radiologic lesion, 11 patients had two radiologic lesions, and two patients had 3 lesions in KUB. Chi square test revealed the significant correlation between radiologic lesions and spinal severity $(P=0.001$, chi square $=25.77)$. By 
Khoshrang $\mathrm{H}$ et al.

\begin{tabular}{|c|c|c|}
\hline Variables & Results & P Value \\
\hline Gender & & 0.07 \\
\hline Male & $72(71.3)$ & \\
\hline Female & $29(28.7)$ & \\
\hline Age, $y$ & $45.5 \pm 12.5$ & 0.48 \\
\hline $15-40$ & $40(39.6)$ & \\
\hline $41-60$ & $48(47.5)$ & \\
\hline$>60$ & $13(12.9)$ & \\
\hline Height, cm & $166.8 \pm 9.5$ & 0.138 \\
\hline$<165$ & $47(46.5)$ & \\
\hline$>165$ & $54(53.5)$ & \\
\hline BMI, $\mathrm{kg} / \mathrm{m}^{2}$ & $25.7 \pm 5.2$ & 0.01 \\
\hline$<20$ & $9(8.9)$ & \\
\hline $20-25$ & $37(36.6)$ & \\
\hline $25-30$ & $41(40.6)$ & \\
\hline$>30$ & $14(13.9)$ & \\
\hline Weight, kg & $71.74 \pm 12.75$ & \\
\hline
\end{tabular}

assessing several correlations between the variables influencing the severity of SA performance, the current study determined that BMI, radiologic lesions, and skeleton deformity of spine are the major factors influencing the difficulty score of SA. Using general linear model (GLM) (nominal logistic regression), the one way ANOVA, and calculating the adjusted odds ratio, likelihood ratio for these variables and the proportional formulation on the basis of the lowest odds ratio, specific coefficients for variables were found:

1) Body mass index (BMI) over 30 was three and no score for BMI less than 30 .

2) The score of radiologic signs of spine in KUB (osteophites, ligament calcification, and reduced intervertebral space) was four and no score for patients without radiologic lesions.

3) The score of skeletal deformity (lordosis, kyphosis, and scoliosis) in spine was three and no score for patients without skeleton deformity.

4) The score of difficulty to find spinal process, neither visible nor palpable, was 10 and no score in easy anatomic landmarks.

With these variables, the current study was able to predict difficulty of SA. By the Nominal Regression, different sums of these scores were obtained. In order to find the cutoff point to predict the difficulty of SA, these variables were used in ROC curve. With maximum specificity, sensitivity, using ROC curve, and cutoff point the spinal severitywas estimated $\geq 8.5$, and spinal severity would happen with a specificity of $86 \%$ and a sensitivity of $86.7 \%$.

\begin{tabular}{lc}
\hline \multicolumn{2}{l}{ Table 2. Difficulty of Spinal Anesthesia in Patients ${ }^{\text {a }}$} \\
\hline Grading & Results \\
\hline Easy & $50(49.5)$ \\
\hline First attempt & $19(18.9)$ \\
\hline Moderate & $17(16.8)$ \\
\hline First space with redirection & \\
\hline Attempt in second space & $8(7.9)$ \\
\hline Difficult & $6(5.9)$ \\
\hline Redirection in second space & $1(0.9)$ \\
\hline Attempt in third space & \\
\hline $\begin{array}{l}\text { Incomplete anesthesia and need to } \\
\text { other analgesic agents }\end{array}$ & $0(0)$ \\
\hline $\begin{array}{l}\text { Failure to complete block } \\
\text { Total }\end{array}$ & $101(100)$ \\
\hline a Data are presented as No.(\%).
\end{tabular}

Table 3. Difficulty of Spinal Anesthesia in Different Spinal Process Conditions ${ }^{\text {a }}$

\begin{tabular}{|c|c|c|c|c|c|}
\hline & Easy & Moderate & Difficult & Total & P Value \\
\hline Visible & $27(71.1)$ & $10(26.3)$ & $1(2.6)$ & $38(37.6)$ & 0.01 \\
\hline Palpable & $21(47.7)$ & $20(45.5)$ & $3(6.8)$ & $44(43.6)$ & \\
\hline $\begin{array}{l}\text { Invisible and } \\
\text { impalpable }\end{array}$ & $2(10.5)$ & $6(31.6)$ & $11(9.5)$ & $19(18.8)$ & \\
\hline Total & $50(49.5)$ & $36(35.6)$ & 15 (14.9) & $101(100)$ & \\
\hline
\end{tabular}

a Data are presented as No. (\%).

Table 4. Distribution of Spinal Anesthesia Difficulty by Body Mass Index (BMI) levels ${ }^{\text {a,b }}$

\begin{tabular}{|c|c|c|c|c|c|}
\hline BMI, $\mathrm{kg} / \mathrm{m}^{2}$ & Easy & Moderate & Difficult & Total & PValue \\
\hline$<\mathbf{2 0}$ & $4(44.4)$ & $4(44.4)$ & 1(11.1) & $9(100)$ & 0.01 \\
\hline 20-25 & $21(56.8)$ & $12(32.4)$ & $4(10.8)$ & $37(100)$ & \\
\hline $25-30$ & $22(53.7)$ & $15(36.6)$ & $4(9.8)$ & $41(100)$ & \\
\hline$>30$ & $3(21.4)$ & $5(35.7)$ & $6(42.9)$ & $14(100)$ & \\
\hline
\end{tabular}

a Abbreviations: BMI, body mass index.

$\mathrm{b}$ Data are presented as No. (\%).

\section{Discussion}

The current study showed a meaningful correlation between BMI, skeletal spinal deformity, radiologic lesion, spinous process condition, and difficulty score for SA that can be used as predicting factors to determine the difficulty score for SA. Results of the study by Atallah et al. on300 patients showed that spinal process condition and radiologic signs of vertebra were two important predicting factors of difficult SA, but anesthesiologistl experiences had no impact on spinal severity (8). The results of their study confirmed the results of the current study on radiologic lesion ( $\mathrm{P}=0.001)$, and spinous process condition ( $P=0.001)$ that can affect difficult SA. (8). In 1999, 
a study conducted by Spurg et al. showed that spine anatomic features had the most impact on spinal severity, and body habitus influenced the frequency of attempts for spinal puncture. In this study age, gender, needle size and anesthesiologist experience had no effect on spinal severity (12). In the current study age, gender and height had no effect on difficulty score of SA and for all patients the same type and size of needle was used and the same anesthesiologist performed the procedure. In the current study, success rate for accurate identification of the subarachnoid space on the first skin puncture (49.5\%) was lower compared to SA performances under real-time ultrasound guidance (9). In the study by Ellinas et al., 427 pregnant patients were evaluated. They found that the practitioner's ability was the most significant predictor of difficulty in SA but BMI was not an independent predictor of either end points (13). In the current study, t-test $(P=0.138)$ and Chi-square test $(P=0.346)$ showed no significant difference between the height and spinal severity and the one way ANOVA parametric test showed a significant correlation between BMI and spinal severity $(P=0.068)$, and Pearson correlation coefficient confirmed this result $(\mathrm{P}=0.004$, Pearson correlation $=$ 0.286 ), which meant there was a correlation between the increased BMI and an increase in difficulty of SA. Hebl et al. (14) reported that the history of past spinal surgery could not affect spinal severity, which would confirm the current study results. Since in the current study there was only one patient with the history of spinal surgery, its effects on difficulty score of SA were not interpretable. Garg et al. concluded that in patients with ligament calcification there would be a need of introducer for spinal needle (15). In the current study radiologic features (ligament calcifications, osteophites and reduced intervertebral space) had significant correlation with difficulty score of SA. $(\mathrm{P}=0.001)$. In the study by Gupta et al. more failures in SA occurred in kyphoscoliosis patients and resulted in more failures of spinal anesthesia, which was more common in patients with a past spinal surgery. Their complications caused failure in SA and incomplete anesthesia (16). The current study results showed that BMI, radiologic lesions and skeletal deformity of spine were effective factors in difficulty score of SA. Spinous process condition was the most important factor to predict spinal severity and if patients had this complication, they needed more time for anesthesia process leading to prolonged anesthesia time; relocation of the needle and more punctures would be needed leading to headache and backache resulting the patients dissatisfaction. Providing a scoring system which quantifies difficulty score of SA could help the anesthesiologist to predict difficulty or failure of spinal anesthesia and would help him to choose the best technique that would match the patients' condition. This scoring is also helpful in emergency cases like fetal distress and a need of emergency cesarean section. It seems that although the radiologic study of spine is not required in all patients if for any reasons the patient has this radiographs (trauma or urologic operation), spine radiographs would be a valuable help to predict difficulty score for SA. In the current study, the concluded results concerning the patients' physical examination focusing on lumbar spinous process, skeletal spinal deformity, lumbar radiological findings and BMI can be helpful to select or not to select the spinal anesthesia, and it is also valuable to prevent its side effects.

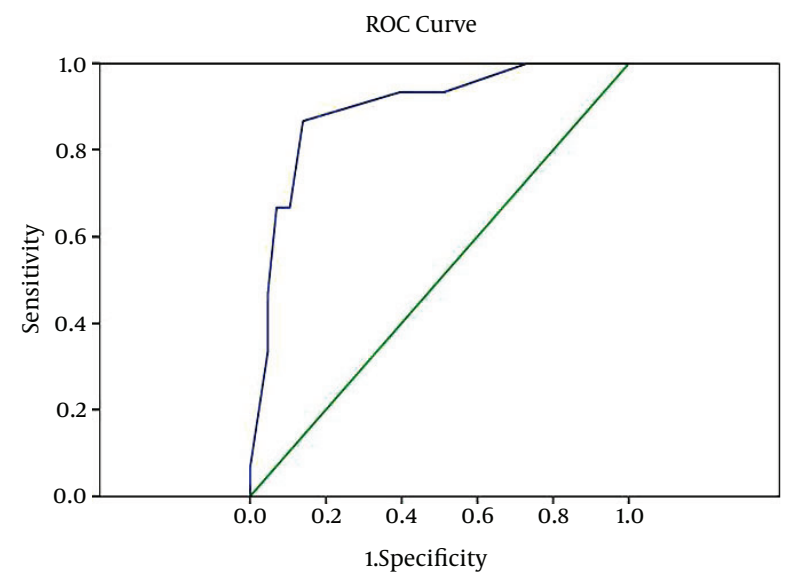

Figure 1. Relative Operating Characteristic Curve to Find the Cut-off Point

\section{Acknowledgements}

Authors acknowledge their gratitude to the Urology Research Center and Research Deputy of Gilan University of Medical Sciences (GUMS) to help them perform the current project.

\section{Author's Contributions}

Khoshrang H.: Substantial contributions to conception and design, acquisition, interpretation or analysis of data; drafting the article or critical revising; final approval of the manuscript. Falahatkar S.: Substantial contributions to conception and design, drafting the article or critical revising; final approval of the manuscript. Heidarzadeh A.: Acquisition of data, or analysis and interpretation of data. Abad M.: Substantial contributions to conception and design, acquisition, interpretation or analysis of data; drafting the article or critical revising. Rastjou Herfeh N.: Drafting the article or critical revising; final approval of the manuscript. Naderi Nabi B.: Drafting the article or critical revising.

\section{References}

1. Chien I, Lu IC, Wang FY, Soo LY, Yu KL, Tang CS. Spinal process landmark as a predicting factor for difficult epidural block: a prospective study in Taiwanese patients. Kaohsiung J Med Sci. 2003;19(11):563-8.

2. Zundert AV, Bruinsma A. Annemarieke Bruinsma. Neurological 
complications of central neuraxial blockade. ELIPSA-JAIM s.c.; 2009.

3. Rhee WJ, Chung CJ, Lim YH, Lee KH, Lee SC. Factors in patient dissatisfaction and refusal regarding spinal anesthesia. Korean J Anesthesiol.2010;59(4):260-4.

4. Falahatkar S, Khosropanah I, Allahkhah A, Jafari A. Open surgery, laparoscopic surgery, or transureteral lithotripsy--which method? Comparison of ureteral stone management outcomes. J Endourol. 2011;25(1):31-4.

5. Nikoobakht MR, Emamzadeh A, Abedi AR, Moradi K, Mehrsai A. Transureteral lithotripsy versus extracorporeal shock wave lithotripsy in management of upper ureteral calculi: a comparative study. Urol J. 2007;4(4):207-11.

6. Paik ML, Wainstein MA, Spirnak JP, Hampel N, Resnick MI. Current indications for open stone surgery in the treatment of renal and ureteral calculi. JUrol.1998;159(2):374-8.

7. Boon JM, Abrahams PH, Meiring JH, Welch T. Lumbar puncture: anatomical review of a clinical skill. Clin Anat. 2004;17(7):544-53.

8. Atallah MM, Demian AD, Shorrab AA. Development of a difficulty score for spinal anaesthesia. BrJ Anaesth. 2004;92(3):354-60.

9. Conroy PH, Luyet C, McCartney CJ, McHardy PG. Real-time ultrasound-guided spinal anaesthesia: a prospective observational study of a new approach. Anesthesiol Res Pract. 2013;2013:525818.

10. Fettes PD, Jansson JR, Wildsmith JA. Failed spinal anaesthesia: mechanisms, management, and prevention. Br J Anaesth. 2009;102(6):739-48.

11. Weed J, Finkel K, Beach ML, Granger CB, Gallagher JD, Sites BD. Spinal anesthesia for orthopedic surgery: a detailed video assessment of quality. Reg Anesth Pain Med. 2011;36(1):51-5.

12. Sprung J, Bourke DL, Grass J, Hammel J, Mascha E, Thomas P, et al. Predicting the difficult neuraxial block: a prospective study. Anesth Analg. 1999;89(2):384-9.

13. Ellinas EH, Eastwood DC, Patel SN, Maitra-D'Cruze AM, Ebert TJ. The effect of obesity on neuraxial technique difficulty in pregnant patients: a prospective, observational study. Anesth Analg. 2009;109(4):1225-31.

14. Hebl JR, Horlocker TT, Kopp SL, Schroeder DR. Neuraxial blockade in patients with preexisting spinal stenosis, lumbar disk disease, or prior spine surgery: efficacy and neurologic complications. Anesth Analg. 2010;111(6):1511-9.

15. Garg R, Kumar A, Pandey R. Deformed spinal needle causing PDPH and dry tap due to blood clot. Local Reg Anesth. 2010;3:27-9.

16. Gupta S, Singariya G. Kyphoscoliosis and pregnancy-A case report. Indian J Anaesth. 2004;48(3):215-20. 